\title{
Axion Dark Matter Search with Interferometric Gravitational Wave Detectors
}

\author{
Koji Nagano $\odot,{ }^{1}$ Tomohiro Fujita, ${ }^{2,3}$ Yuta Michimura, ${ }^{4}$ and Ippei Obata ${ }^{1}$ \\ ${ }^{1}$ Institute for Cosmic Ray Research, University of Tokyo, Kashiwa 277-8582, Japan \\ ${ }^{2}$ Department of Physics, Kyoto University, Kyoto, 606-8502, Japan \\ ${ }^{3}$ Départment de Physique Théorique and Center for Astroparticle Physics, Université de Genève, \\ Quai E.Ansermet 24, CH-1211 Genève 4, Switzerland \\ ${ }^{4}$ Department of Physics, University of Tokyo, Bunkyo, Tokyo 113-0033, Japan
}

(Received 2 April 2019; revised manuscript received 2 July 2019; published 13 September 2019)

\begin{abstract}
Axion dark matter differentiates the phase velocities of the circular-polarized photons. In this Letter, a scheme to measure the phase difference by using a linear optical cavity is proposed. If the scheme is applied to the Fabry-Pérot arm of Advanced-LIGO-like (Cosmic-Explorer-Like) gravitational wave detector, the potential sensitivity to the axion-photon coupling constant, $g_{\mathrm{a} \gamma}$, reaches $g_{\mathrm{a} \gamma} \simeq 8 \times 10^{-13} \mathrm{GeV}^{-1}\left(4 \times 10^{-14} \mathrm{GeV}^{-1}\right)$ at the axion mass $m \simeq 3 \times 10^{-13} \mathrm{eV}\left(2 \times 10^{-15} \mathrm{eV}\right)$ and remains at around this sensitivity for three orders of magnitude in mass. Furthermore, its sensitivity has a sharp peak reaching $g_{\mathrm{a} \gamma} \simeq$ $10^{-14} \mathrm{GeV}^{-1}\left(8 \times 10^{-17} \mathrm{GeV}^{-1}\right)$ at $m=1.563 \times 10^{-10} \mathrm{eV}\left(1.563 \times 10^{-11} \mathrm{eV}\right)$. This sensitivity can be achieved without losing any sensitivity to gravitational waves.
\end{abstract}

DOI: 10.1103/PhysRevLett.123.111301

Introduction.-The axion is a pseudoscalar field that was originally proposed in the late 1970 s to solve the strong $C P$ problem in QCD physics, known as a "QCD axion" [1]. In recent decades, it has been found that high energy physics such as string theory also predicts a number of axionlike particles from the compactification of extra dimensions [2]. Hereafter we collectively call them "axions." An axion typically has a small mass, $m \ll \mathrm{eV}$, and behaves as like a nonrelativistic fluid in the present Universe due to its oscillatory behavior. For this reason, an axion is a cosmologically well-motivated candidate of dark matter. Another important feature of an axion is its coupling to gauge bosons. In particular, a small but finite coupling between an axion and a photon is a general prediction of high energy physics and provides a good chance to detect an axion by using the well-developed photonics technology.

The conventional way to probe axions is to look for a phenomena where an axion and a photon convert each other under the background magnetic field, known as the axionphoton conversion [3]. Many experiments and astronomical observations have been performed to probe an axion via the axion-photon conversion [4-22], while no signal has been found (for recent reviews, see Ref. [23]). Recently, however, a new experimental approach to search for axion dark matter was proposed that does not need a strong magnetic

Published by the American Physical Society under the terms of the Creative Commons Attribution 4.0 International license. Further distribution of this work must maintain attribution to the author(s) and the published article's title, journal citation, and DOI. Funded by SCOAP ${ }^{3}$. field but uses optical cavity [24-27]. This new method aims to measure the difference of phase velocity between two circular-polarized photons, which is caused by the coupling to axion dark matter $[28,29]$. The experimental sensitivity is only limited by quantum noise in principle and it can probe tiny axion-photon coupling $g_{\mathrm{a} \gamma} \lesssim 10^{-11} \mathrm{GeV}^{-1}$ with axion mass range $m \lesssim 10^{-10} \mathrm{eV}$ which is competitive with other experimental proposals. Moreover, this new method can be highly advantageous compared with the conventional axion detectors, since it does not require superconducting magnets, which often involve a large cost. Therefore, we expect that this method will open a new window to the axion dark matter research.

Inspired by these proposals using optical cavity, in this Letter we propose a new scheme to search for axion dark matter by using a linear Fabry-Pérot cavity. Linear optical cavities are used in the current and future gravitational wave detectors, such as Advanced LIGO (aLIGO) [30], Advanced Virgo [31], KAGRA [32], Einstein Telescope [33], Cosmic Explorer (CE) [34], and DECIGO [35]. In this Letter, we explore the capabilities of these laser interferometers to search for axionlike dark matter. Remarkably, our new method enables the interferometers to probe axionlike dark matter during the gravitational wave observation run without losing any sensitivity to gravitational waves. It implies that we can exploit the cutting-edge laser facilities for axionlike dark matter search even without constructing a dedicated one from scratch. We estimate the potential sensitivity to the axion-photon coupling with the parameter sets of gravitational wave observatories. Their sensitivities can overcome the current upper limit with broad axion mass range and put better bounds than the 
proposed axion experiments by several orders of magnitudes. Note that although the gravitational wave detectors are discussed, we do not propose to measure gravitational waves. Our target is the phase velocity difference in circular-polarized photons, and a laser interferometer is suitable for its detection. Thus, our proposal is complementary to the previous study of the gravitational waves sourced by axions [36].

This Letter is organized as follows. In the next section, we shortly derive the difference in the phase velocity of polarized photons in the presence of axion dark matter. Then we present the scheme to detect it as a polarization modulation of a linearly polarized light using the FabryPérot cavity to enhance the modulation. Next we describe the prospected sensitivity curves of axion-photon coupling with each gravitational wave interferometer. Finally, we give a short discussion and conclude with our results. In this Letter, we set the natural unit $\hbar=c=1$.

Phase velocity modulation.-In this section, we briefly explain how the dispersion relations of two circularpolarized photons are modified in the presence of background axion field. The axion-photon coupling is written as a Chern-Simons interaction

$\frac{g_{\mathrm{a} \gamma}}{4} a(t) F_{\mu \nu} \tilde{F}^{\mu \nu}=g_{\mathrm{a} \gamma} \dot{a}(t) \epsilon_{i j k} A_{i} \partial_{j} A_{k}+($ total derivative $)$,

where the dot denotes the time derivative, $g_{\mathrm{ay}}$ is a coupling constant, $a(t)$ is the axion field value, and $A_{\mu}$ is the vector potential of the electromagnetic field strength $F_{\mu \nu} \equiv \partial_{\mu} A_{\nu}-$ $\partial_{\nu} A_{\mu}$. Its Hodge dual is defined as $\tilde{F}^{\mu \nu} \equiv \epsilon^{\mu \nu \rho \sigma} F_{\rho \sigma} / 2$, where $\epsilon^{\mu \nu \rho \sigma}$ is the Levi-Civita antisymmetric tensor. Regarding the gauge condition, we choose the temporal gauge $A_{0}=0$ and the Coulomb gauge $\partial_{i} A_{i}=0 . A_{i}$ can be decomposed into two circular polarization modes in the Fourier space

$$
A_{i}(t, \boldsymbol{x})=\sum_{\lambda=L, R} \int \frac{d^{3} k}{(2 \pi)^{3}} A_{\lambda}(t, \boldsymbol{k}) e_{i}^{\lambda}(\hat{\boldsymbol{k}}) e^{i \boldsymbol{k} \cdot \boldsymbol{x}},
$$

where $\boldsymbol{k}$ is the wave number vector, the circular polarization vectors satisfy $e_{i}^{\lambda}(\hat{\boldsymbol{k}})=e_{i}^{\lambda *}(-\hat{\boldsymbol{k}}), e_{i}^{\lambda}(\hat{\boldsymbol{k}}) e_{i}^{\lambda^{\prime} *}(\hat{\boldsymbol{k}})=\delta^{\lambda \lambda^{\prime}}$ and $i \epsilon_{i j m} k_{j} e_{m}^{L / R}(\hat{\boldsymbol{k}})= \pm k e_{i}^{L / R}(\hat{\boldsymbol{k}})(k \equiv|\boldsymbol{k}|)$. Here the index of $L(R)$ corresponds to the upper (lower) sign of the double sign. Hereafter, we use the same notation in this Letter. Then the equation of motion for $A_{L / R}(t, \boldsymbol{k}), \ddot{A}_{L / R}+\omega_{L / R}^{2} A_{L / R}=0$, acquires the modified dispersion relation due to the axionphoton coupling Eq. (1), $\omega_{L / R}^{2}=k^{2}\left(1 \mp g_{\mathrm{a} \gamma} \dot{a} / k\right)$. This leads to the different phase velocities for the left and right polarization modes

$$
c_{L / R}^{2}=1 \mp \frac{g_{\mathrm{a} \gamma} \dot{a}}{k} .
$$

Note that the momentum effect of axion dark matter here is irrelevant since it is nonrelativistic. Ignoring the cosmic expansion, the present axion dark matter is given by the periodic function

$$
a(t)=a_{0} \cos \left[m t+\delta_{\tau}(t)\right],
$$

with the frequency of axion mass $f=m /(2 \pi) \simeq 2.4 \mathrm{~Hz}$ $\left(m / 10^{-14} \mathrm{eV}\right)$. The phase factor $\delta_{\tau}(t)$ can be regarded as a constant value within the coherent timescale of axion dark matter, $\tau$, expressed as $\tau=2 \pi /\left(m v_{\mathrm{a}}^{2}\right)$, where $v_{\mathrm{a}}$ is an axion dark matter velocity. Since the local velocity of dark matter is about $10^{-3}, \tau$ is estimated as

$$
\tau \sim 1\left(\frac{10^{-16} \mathrm{eV}}{m}\right) \text { year. }
$$

Plugging Eq. (4) into Eq. (3), we obtain

$$
c_{L / R}(t) \simeq 1 \pm \delta c(t) \equiv 1 \pm \delta c_{0} \sin \left[m t+\delta_{\tau}(t)\right],
$$

where $\delta c_{0}=g_{\mathrm{a} y} a_{0} m /(2 k)$ is the maximum difference of the phase velocity, $c_{0}$ is the speed of light without background axion, and $\delta c_{0} \ll 1$ is used. $\delta c_{0}$ is estimated as

$$
\delta c_{0} \simeq 1.3 \times 10^{-24}\left(\frac{\lambda}{1550 \mathrm{~nm}}\right)\left(\frac{g_{\mathrm{a \gamma}}}{10^{-12} \mathrm{GeV}^{-1}}\right) .
$$

Here we assumed the laser light with a wavelength $\lambda=2 \pi / k$ and used the present energy density value of an axion dark matter around earth, $\rho_{a}=a_{0}^{2} m^{2} / 2 \simeq 0.3 \mathrm{GeV} / \mathrm{cm}^{3}$, which removes the dependence of $\delta c_{0}$ on $a_{0} m$.

The key point is that, according to the Eq. (6), one linearly polarized light (e.g., horizontal polarization, that is $p$ polarization) is polarization modulated due to an axion dark matter and the orthogonally polarized light (e.g., vertical polarization, that is $s$ polarization) is produced, as shown later. Note that the linearly polarized light can be expressed by a superposition of two circularly polarized lights. From the next section, we show that this polarization-modulation can be measured with linear cavities of gravitational wave experiments by using our proposed method.

Axion search with a linear optical cavity.-In this section, we present how to detect the modulation of speed of light with linear optical cavities. The schematic setup of our proposed scheme is shown in Fig. 1. First, as a carrier wave, we input linearly polarized monochromatic laser light with the angular frequency that corresponds to the wave number $k$. Here, we consider $p$-polarized light as input light without losing generality. The cavity consists of the input and output mirrors whose amplitude reflectivities and transmissivities are represented by $\left(r_{1}, t_{1}\right)$ and $\left(r_{2}, t_{2}\right)$. In this Letter, we only consider the axion mass range where $\tau$ is longer than the cavity storage time, $4 \pi L \sqrt{r_{1} r_{2}} /$ $\left(1-r_{1} r_{2}\right)$ [37]. In this condition, the axion can be treated 


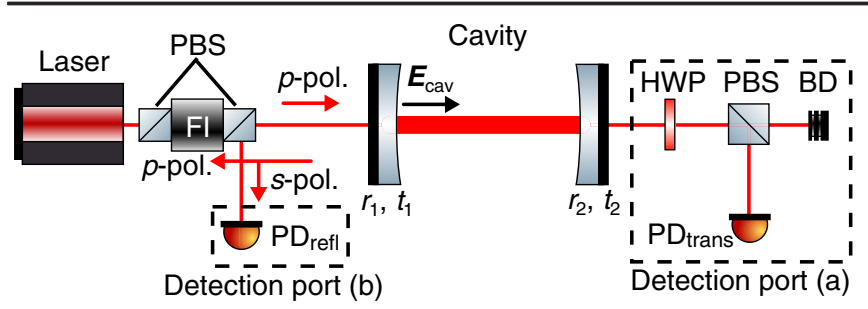

FIG. 1. Schematic of experimental setup for the axion search with a linear optical cavity. FI, Faraday isolator; HWP, half wave plate; PBS, polarizing beam splitter; PD, photodetector; BD, beam dump. Signal is detected in detection port (a) and (b). Components for phase measurement are not shown. The polarization of incident light is arbitrary only if it is linear polarization. Two PBSs in FI are placed, rotated by 45 degrees, along the optical path.

as a coherent oscillator during the time when photon is interacting with the axion in the cavity. When the cavity is kept to resonate with a phase measurement, such as PoundDrever-Hall technique [38], the beam is accumulated inside a cavity, and the signal, $\delta c$, is enhanced as explained later. Then the signal is detected in detection port (a) or (b) as the polarization modulation with polarizing optics. In detection port (a), the polarization of transmitted light from the cavity is slightly rotated by the half wave plate. Then, the photodetector $\left(\mathrm{PD}_{\text {trans }}\right)$ receives $s$-polarized light generated by axion-photon coupling as a beat note with a faint (but much stronger than the signal) carrier wave, while most of the carrier light is transmitted by the polarizing beam splitter (PBS). In detection port (b), the PD ( $\left.\mathrm{PD}_{\text {refl }}\right)$ receives signal reflected by the faraday isolator (FI) as a beat note with a faint carrier wave again. In this case, the carrier wave is generated by nonideal birefringence between the cavity and FI, such as input mirror substrate. These two detection ports can be added without modifying the instrument for the phase measurement.

The signal, $\delta c$, is enhanced inside the cavity by the following mechanism. Here, we treat $\delta_{\tau}(t)$ as a constant since we only consider the axion mass range where the axion oscillation coherent time is sufficiently longer than the storage time of the optical cavity. The input $p$-polarized light is written as

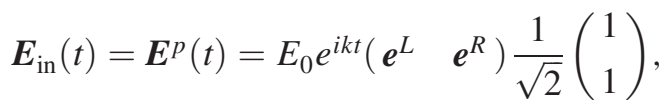

where $\boldsymbol{E}^{p}(t)$ is the electric vector of $p$-polarized light, $\boldsymbol{e}^{L}$ and $\boldsymbol{e}^{R}$ are basis vectors of left-handed and right-handed laser light, respectively. In the presence of background axion field, the electric vector propagation in the cavity in front of the front mirror is expressed as

$$
\boldsymbol{E}_{\mathrm{cav}}(t)=t_{1} E_{0} e^{i k t}\left(\boldsymbol{e}^{L} \boldsymbol{e}^{R}\right) \sum_{n=1}^{\infty} A_{n}(t) \frac{1}{\sqrt{2}}\left(\begin{array}{l}
1 \\
1
\end{array}\right),
$$

$$
\begin{array}{ll}
A_{n+1}(t) \equiv & A_{n}(t) R_{1} T[t-2 L(n-1)] \\
& \times R_{2} T[t-2 L(n-1 / 2)] \quad(n \geq 1),
\end{array}
$$

where $L$ is cavity length, $T(t)$ is transfer matrix for one-way translation,

$$
\begin{gathered}
T(t) \equiv\left(\begin{array}{cc}
e^{-i \phi^{\mathrm{L}}(t)} & 0 \\
0 & e^{-i \phi^{\mathrm{R}}(t)}
\end{array}\right), \\
\phi^{L / R}(t) \equiv k L \mp k \int_{t-L}^{t} \delta c\left(t^{\prime}\right) d t^{\prime},
\end{gathered}
$$

and $R_{i}$ is the reflection matrix for circularly polarized lights,

$$
R_{i} \equiv\left(\begin{array}{cc}
0 & -r_{i} \\
-r_{i} & 0
\end{array}\right) \quad(i=1,2)
$$

Sign flipping in Eq. (13) is the main difference from the modeling in Refs. [25-27]. Here $A_{n}(t)(n \geq 2)$ is given by,

$$
A_{n}(t)=\left(r_{1} r_{2}\right)^{n-1}\left(\begin{array}{cc}
A_{n}^{11}(t) & 0 \\
0 & A_{n}^{22}(t)
\end{array}\right),
$$

with

$$
\begin{aligned}
A_{n}^{11 / 22} \equiv & \exp [-i k\{2 L(n-1) \\
& \left.\left. \pm \sum_{j=1}^{n-1}\left(\int_{t-2 L(j-1 / 2)}^{t-2 L(j-1)}-\int_{t-2 L j}^{t-2 L(j-1 / 2)}\right) \delta c\left(t^{\prime}\right) d t^{\prime}\right\}\right]
\end{aligned}
$$

where the 11 and 22 component of $A_{n}$ corresponds to the upper and lower sign of the flipped sign, respectively. When resonance condition of the linear cavity, $2 k L=2 \pi l$ $(l \in \mathbb{N})$, is met, $A_{n}^{11}$ and $A_{n}^{22}$ are also denoted as

$$
\begin{aligned}
A_{n}^{11 / 22}= & \exp \left[\mp i k \int_{-\infty}^{\infty} \widetilde{\delta c}(m) \frac{1}{m} \tan \left(\frac{m L}{2}\right)\right. \\
& \left.\times\left(1-e^{i 2 m L(n-1)}\right) e^{i m t} \frac{d m}{2 \pi}\right],
\end{aligned}
$$

where we transformed $\delta c(t)$ in Fourier space, $\delta c(t)=$ $\int_{-\infty}^{\infty} \widetilde{\delta c}(m) e^{i m t}(d m / 2 \pi)$. Consequently, the electronic field in the cavity is written as, 


$$
\begin{aligned}
\boldsymbol{E}_{\mathrm{cav}}(t)= & \frac{t_{1} E_{0} e^{i k t}}{1-r_{1} r_{2}}\left(\begin{array}{ll}
\boldsymbol{e}^{L} & \boldsymbol{e}^{R}
\end{array}\right) \\
& \times\left(\begin{array}{cc}
1+i \delta \phi(t) & 0 \\
0 & 1-i \delta \phi(t)
\end{array}\right) \frac{1}{\sqrt{2}}\left(\begin{array}{l}
1 \\
1
\end{array}\right) \\
= & \frac{t_{1}}{1-r_{1} r_{2}}\left[\boldsymbol{E}^{p}(t)-\delta \phi(t) \boldsymbol{E}^{s}(t)\right],
\end{aligned}
$$

where $\boldsymbol{E}^{s}$ are electric vectors of $s$-polarized light,

$$
\delta \phi(t) \equiv \int_{-\infty}^{\infty} \widetilde{\delta c}(m) H_{\mathrm{a}}(m) e^{i m t} \frac{d m}{2 \pi},
$$

and $H_{\mathrm{a}}(m)$ is a response function of cavity,

$$
H_{\mathrm{a}}(m) \equiv i \frac{k}{m} \frac{4 r_{1} r_{2} \sin ^{2}\left(\frac{m L}{2}\right)}{1-r_{1} r_{2} e^{-i 2 m L}}\left(-e^{-i m L}\right) .
$$

Equation (20) indicates that the signal is enhanced in proportion to $r_{1} r_{2} /\left(1-r_{1} r_{2}\right)$ at $m=\pi / L$, which corresponds to the free spectral range, i.e., the frequency separation of the longitudinal mode of the cavity [37]. The peak sensitivity can be enhanced by increasing the mirror reflectivity, although finesse, $\pi \sqrt{r_{1} r_{2}} /\left(1-r_{1} r_{2}\right)$, is limited to be lower than $10^{6}$ due to the dispersion of the dark matter [39]. In addition, $H_{\mathrm{a}}(m) \propto 1 / m$ at $m L=$ $\pi(2 N-1)(N \in \mathbb{N})$ since the axion effect on the photons in the cavity is cancelled out except for the last half of the axion oscillation when the axion oscillation period is shorter than the photon storage time of the cavity [37]. In a low mass range $(m L \ll 1), H_{\mathrm{a}}(m) \propto m$ since the axion effect is cancelled on both the going and returning way, due to Eq. (13).

Sensitivity to the axion-photon coupling.-In this section, we estimate the potential sensitivity of the linear cavity to axion-photon coupling. Here, only shot noise, which is caused by vacuum fluctuation of electric field, $E_{\mathrm{vac}}(t)$, is considered in a similar way to the shot-noise estimation of gravitational wave detectors [40]. In each detection port, the electric field received by photodetector is expressed as

$$
\begin{gathered}
\boldsymbol{E}_{\mathrm{PD}}(t)=\left(\sqrt{\mathcal{T}_{j}}[\alpha-\delta \phi(t)]+\frac{E_{\mathrm{vac}}(t)}{E_{0}}\right) \boldsymbol{E}^{\mathrm{s}}(t) \quad(j=1,2), \\
\sqrt{\mathcal{T}_{j}} \equiv \frac{t_{1} t_{j}}{1-r_{1} r_{2}},
\end{gathered}
$$

where $\alpha(|\alpha| \ll 1)$ is the polarization mixing angle introduced by the half wave plate (instrumental birefringence) and $j=2$ (1) for the detection port (a) [(b)]. Here, we neglect the second and higher order of $|\alpha|$. Note that $|\alpha|$ is much larger than $|\delta \phi(t)|$ and $\left|E_{\mathrm{vac}}(t)\right|$. The detected power is

$$
\begin{aligned}
P_{\mathrm{PD}}(t) & \propto\left|\boldsymbol{E}_{\mathrm{PD}}(t)\right|^{2} \\
& \simeq \alpha \sqrt{\mathcal{T}_{j}} E_{0}^{2}\left[\alpha \sqrt{\mathcal{T}_{j}}-2 \sqrt{\mathcal{T}_{j}} \delta \phi(t)+2 \frac{E_{\mathrm{vac}}(t)}{E_{0}}\right],
\end{aligned}
$$

where the second order and cross terms of $\delta \phi(t)$ and $E_{\mathrm{vac}}(t)$ are ignored. We can estimate the sensitivity by comparing the second and third terms of Eq. (23) which are time dependent. The second and third term corresponds to signal and shot noise, respectively. The one-sided linear spectrum of shot noise equivalent to $\widetilde{\delta c}(m), \sqrt{S_{\text {shot }}(m)}$, is obtained by considering the ratio of the noise term to the signal term,

$$
\sqrt{S_{\text {shot }}(m)}=\frac{\sqrt{\frac{k}{2 P_{0}}}}{\sqrt{\mathcal{T}_{j}}\left|H_{\mathrm{a}}(m)\right|},
$$

where $P_{0}$ is incident power. Here, we used $E_{0}=\sqrt{2 P_{0} / k}$, and the one-sided spectrum of vacuum fluctuation is unity [40]. In this Letter, the electric field has dimensions of $[\sqrt{\mathrm{Hz}}]$ as in Ref. [40]. According to Eq. (24), if the cavity is overcoupled, i.e., $t_{1}>t_{2}$, the detection port (b) is better. On the other hand, detection port (a) is effective for the criticalcoupled cavity, i.e., $t_{1}=t_{2}$, since there is no carrier wave in the reflection port under the critical coupling condition.

If the sensitivity is limited by shot noise, the signal-to-noise ratio (SNR) for $\delta c_{0}$ is improved with measurement time, $T_{\mathrm{obs}}$. The improvement depends on whether $T_{\mathrm{obs}}$ is larger than the coherent time of axion oscillation, $\tau$, or not [41]:

$$
\mathrm{SNR}=\left\{\begin{array}{ll}
\frac{\sqrt{T_{\mathrm{obs}}}}{2 \sqrt{S_{\mathrm{shot}}(m)}} \delta c_{0} & \left(T_{\mathrm{obs}} \lesssim \tau\right) \\
\frac{\left(T_{\mathrm{obs}} \tau\right)^{1 / 4}}{2 \sqrt{S_{\text {shot }}(m)}} \delta c_{0} & \left(T_{\mathrm{obs}} \gtrsim \tau\right)
\end{array} .\right.
$$

We can find the detectable value of $\widetilde{\delta c}(m)$ which sets the SNR to unity

$$
\delta c_{0} \simeq\left\{\begin{array}{ll}
\frac{2}{\sqrt{T_{\mathrm{obs}}}} \sqrt{S_{\text {shot }}(m)} & \left(T_{\mathrm{obs}} \lesssim \tau\right) \\
\frac{2}{\left(T_{\mathrm{obs} \tau} \tau\right)^{1 / 4}} \sqrt{S_{\text {shot }}(m)} & \left(T_{\mathrm{obs}} \gtrsim \tau\right)
\end{array} .\right.
$$

Finally, this is translated into the sensitivity to $g_{\mathrm{a}}$ as

$$
\begin{array}{r}
g_{\mathrm{ay}}(m) \simeq 1.5 \times 10^{12} \mathrm{GeV}^{-1}\left(\frac{1550 \mathrm{~nm}}{\lambda}\right) \\
\times\left\{\begin{array}{ll}
\sqrt{\frac{S_{\text {shot }}(m)}{T_{\mathrm{obs}}}} & \left(T_{\mathrm{obs}} \lesssim \tau\right) \\
\sqrt{\frac{\text { shot }_{\mathrm{sh}}}{\sqrt{T_{\mathrm{obs}} \tau}}} & \left(T_{\mathrm{obs}} \gtrsim \tau\right)
\end{array} .\right.
\end{array}
$$

Figure 2 shows the shot-noise limited sensitivities to $g_{\mathrm{ay}}$ with our scheme. Here, we adopted the experimental 


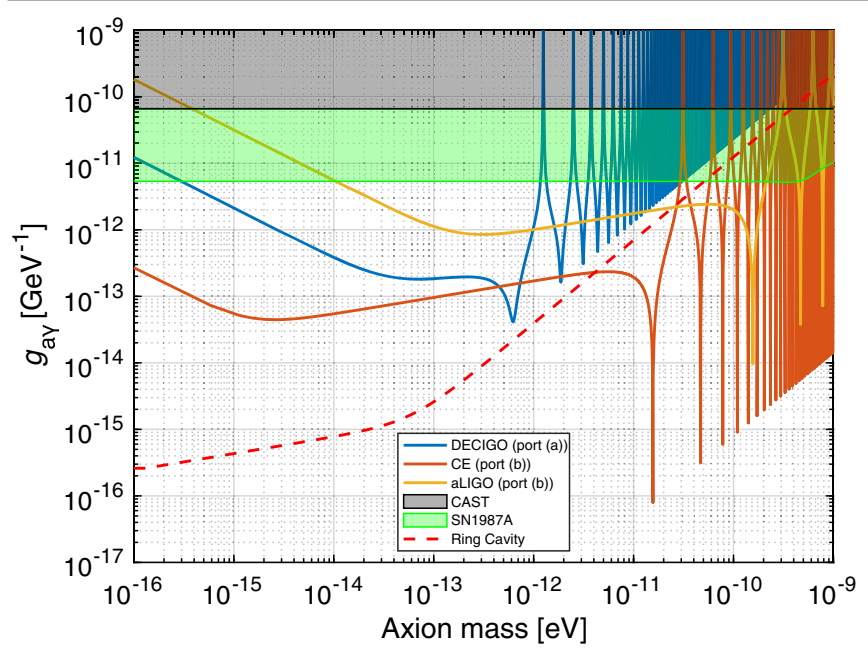

FIG. 2. Sensitivity comparison of the several parameter sets shown in Table I. Although the higher mass range seems to be filled, they have sensitivity peaks at mass of $m=\pi(2 N-1)$ / $L(N \in \mathbb{N})$. The gray and green band express the current limit provided by CAST [5] and the cosmic ray observations of SN1987A [14]. The red dashed line is a sensitivity curve of one proposed experiment using optical ring cavity with optimistic parameters [26].

parameter sets used or planned by gravitational wave detectors (specifically, DECIGO [35], CE [34], and aLIGO [30]) as shown in Table I. We also assume $T_{\mathrm{obs}}=$ 1 year and $r_{i}^{2}+t_{i}^{2}=1$. Note that detection port (a) is used for a DECIGO-like detector and port (b) is used for CE- and aLIGO-like detectors. All gravitational wave detectors have a sensitive mass range similar to some proposed experiment such as IAXO [6] and ABRACADABRA [11]. In all parameter sets, the upper limit provided by CAST [5] can be improved. Especially, the CE-like detector can overcome the CAST limit by three orders of magnitude in broad mass range around between $4 \times 10^{-16}$ and $1 \times 10^{-13} \mathrm{eV}$. At the most sensitive mass $m=1.563 \times 10^{-11} \mathrm{eV}$, the improvement from CAST limits is about six orders of magnitude, although QCD axions cannot be detected.

It is worth noting that in our scheme the displacement noise such as the vibration of mirrors or the gravitational wave signal itself does not become manifest unlike a gravitational wave detector. This is because the displacement noises and gravitational waves make the same phase shift in the two circularly polarized lights propagating in the same path, and this phase shift is cancelled in the measurement of the phase difference between two polarized lights. A major technical noise source in our scheme is a roll motion of the mirrors which would generate relative phase shift in the two polarized lights through birefringence of the mirror coating. The effect of the substrate birefringence is relatively small since the signal is enhanced in the cavity. When the laser polarization and coating axis are almost aligned, the noise spectrum is expressed as $\sqrt{S_{\text {roll }}} \simeq$ $\delta \alpha \lambda \theta_{\mathrm{bi}} / L$, where $\delta \alpha$ is roll motion spectrum and $\theta_{\mathrm{bi}}$ is a coating birefringence. In the aLIGO case, $\theta_{\mathrm{bi}} \simeq 10 \mu \mathrm{rad}$ [43]. The seismic motion make $\delta \alpha<10^{-11} \mathrm{rad} / \mathrm{Hz}^{1 / 2}$ for $m>10^{-14} \mathrm{eV}$ if we conservatively assume that coupling from vertical to roll motion is unity [44]. Thus, $\sqrt{S_{\text {roll }}}<$ $3 \times 10^{-26} 1 / \mathrm{Hz}^{1 / 2}$, which is smaller than shot noise level. In DECIGO and CE, the roll motion of the mirror would be small since they would be in space or underground site while aLIGO is on the ground.

In order to apply our method to the real gravitational detector, some optics are added for detection port, and there exist constructional problems. The approach to detect the signal in detection port (b) is not quite simple because there have been equipped several apparatuses, such as a beam splitter, a signal recycling mirror $[45,46]$, and so on, between the front mirror and FI. In principle, the axion signal can be extracted behind the signal recycling mirror as with the gravitational wave signal readout [47]. More practical issues will be investigated in a future work.

Conclusion.-We developed the experimental scheme to search for axionlike dark matter with the optical linear cavity used in gravitational wave detectors. Our experiment measures the production of the linear polarization component opposite to the intrinsic polarization of the incident laser beam caused by the axion-photon coupling. The experimental sensitivity is in principle limited only by quantum shot noise, and other kind of technical disturbances are irrelevant. We estimated the potential sensitivity of detectors to the axion-photon coupling in a broad mass range $10^{-16} \mathrm{eV} \lesssim m \lesssim 10^{-9} \mathrm{eV}$ with the experimental parameters of existing gravitational wave detector projects, such as DECIGO, CE, and aLIGO. As a result, we found that their sensitivities can reach beyond the current limit of CAST [5] with a wide axion mass range and can be competitive with other experimental proposals that were recently suggested [25-27]. Remarkably, our new scheme for axionlike dark matter search can be performed with a

TABLE I. Parameters of considered gravitational wave detectors. Note that $P_{0}$ is the input beam power to front mirror enhanced by the power recycling cavity for aLIGO-like and CE-like detectors [42].

\begin{tabular}{lcccc}
\hline \hline Similar detector & $L[\mathrm{~m}]$ & $P_{0}[\mathrm{~W}]$ & $\lambda\left[\times 10^{-9} \mathrm{~m}\right]$ & $\left(t_{1}^{2}, t_{2}^{2}\right)[\mathrm{ppm}]$ \\
\hline DECIGO [35] & $10^{6}$ & 5 & 515 & $\left(3.1 \times 10^{5}, 3.1 \times 10^{5}\right)$ \\
CE [34] & $4 \times 10^{4}$ & 600 & 1550 & $\left(1.2 \times 10^{3}, 5\right)$ \\
aLIGO [30] & $4 \times 10^{3}$ & 2600 & 1064 & $\left(1.4 \times 10^{4}, 5\right)$ \\
\hline \hline
\end{tabular}


minor modification of the gravitational wave detector and coexist with its observation run for gravitational waves. We expect that this scheme becomes a new approach to search for axion dark matter.

In this work, K. N., Y. M., and T. F. are supported by the JSPS KAKENHI Grant No. JP17J01176, JSPS Grant-inAid for Scientific Research (B) No. 18H01224, and Grant-in-Aid for JSPS Research Fellow No. 17J09103, respectively.

[1] R. D. Peccei and H. R. Quinn, Phys. Rev. Lett. 38, 1440 (1977); S. Weinberg, Phys. Rev. Lett. 40, 223 (1978); F. Wilczek, Phys. Rev. Lett. 40, 279 (1978).

[2] P. Svrcek and E. Witten, J. High Energy Phys. 06 (2006) 051; A. Arvanitaki, S. Dimopoulos, S. Dubovsky, N. Kaloper, and J. March-Russell, Phys. Rev. D 81, 123530 (2010); L. Visinelli and S. Vagnozzi, Phys. Rev. D 99, 063517 (2019); J. P. Conlon, J. High Energy Phys. 05 (2006) 078.

[3] P. Sikivie, Phys. Rev. Lett. 51, 1415 (1983); M. B. Schneider, F. P. Calaprice, A. L. Hallin, D. W. MacArthur, and D. F. Schreiber, Phys. Rev. Lett. 52, 695 (1984); G. Raffelt and L. Stodolsky, Phys. Rev. D 37, 1237 (1988).

[4] C. Hagmann et al. (ADMX Collaboration), Phys. Rev. Lett. 80, 2043 (1998); S. Moriyama, M. Minowa, T. Namba, Y. Inoue, Y. Takasu, and A. Yamamoto, Phys. Lett. B 434, 147 (1998); T. M. Shokair et al., Int. J. Mod. Phys. A 29, 1443004 (2014); B. T. McAllister, G. Flower, E. N. Ivanov, M. Goryachev, J. Bourhill, and M. E. Tobar, Phys. Dark Universe 18, 67 (2017); D. Horns, J. Jaeckel, A. Lindner, A. Lobanov, J. Redondo, and A. Ringwald, J. Cosmol. Astropart. Phys. 04 (2013) 016; A. Caldwell, G. Dvali, B. Majorovits, A. Millar, G. Raffelt, J. Redondo, O. Reimann, F. Simon, and F. Steffen (MADMAX Working Group), Phys. Rev. Lett. 118, 091801 (2017).

[5] K. Zioutas et al. (CAST Collaboration), Phys. Rev. Lett. 94, 121301 (2005); V. Anastassopoulos et al. (CAST Collaboration), Nat. Phys. 13, 584 (2017).

[6] J. K. Vogel et al., arXiv:1302.3273.

[7] K. Ehret et al. (ALPS Collaboration), Nucl. Instrum. Methods Phys. Res., Sect. A 612, 83 (2009); R. Bähre et al., J. Instrum. 8, T09001 (2013).

[8] M. Betz, F. Caspers, M. Gasior, M. Thumm, and S. W. Rieger, Phys. Rev. D 88, 075014 (2013).

[9] H. Tam and Q. Yang, Phys. Lett. B 716, 435 (2012).

[10] P. Sikivie, N. Sullivan, and D. B. Tanner, Phys. Rev. Lett. 112, 131301 (2014).

[11] Y. Kahn, B. R. Safdi, and J. Thaler, Phys. Rev. Lett. 117, 141801 (2016).

[12] M. Silva-Feaver et al., IEEE Trans. Appl. Supercond. 27, 1 (2017).

[13] J. W. Brockway, E. D. Carlson, and G. G. Raffelt, Phys. Lett. B 383, 439 (1996); J. A. Grifols, E. Massó, and R. Toldrà, Phys. Rev. Lett. 77, 2372 (1996).

[14] A. Payez, C. Evoli, T. Fischer, M. Giannotti, A. Mirizzi, and A. Ringwald, J. Cosmol. Astropart. Phys. 02 (2015) 006.
[15] D. Wouters and P. Brun, Phys. Rev. D 86, 043005 (2012); D. Wouters and P. Brun, Astrophys. J. 772, 44 (2013); A. Abramowski et al. (H.E.S.S. Collaboration), Phys. Rev. D 88, 102003 (2013); J. P. Conlon, A. J. Powell, and M. C. David Marsh, Phys. Rev. D 93, 123526 (2016); M. Ajello et al. (Fermi-LAT Collaboration), Phys. Rev. Lett. 116, 161101 (2016); M. Berg, J. P. Conlon, F. Day, N. Jennings, S. Krippendorf, A. J. Powell, and M. Rummel, Astrophys. J. 847, 101 (2017).

[16] M. C. D. Marsh, H. R. Russell, A. C. Fabian, B. R. McNamara, P. Nulsen, and C. S. Reynolds, J. Cosmol. Astropart. Phys. 12 (2017) 036.

[17] J. P. Conlon, F. Day, N. Jennings, S. Krippendorf, and F. Muia, Mon. Not. R. Astron. Soc. 473, 4932 (2018).

[18] F. Aharonian et al. (H.E.S.S Collaboration), Astron. Astrophys. 475, L9 (2007); J. Albert et al. (MAGIC Collaboration), Science 320, 1752 (2008); A. De Angelis, M. Roncadelli, and O. Mansutti, Phys. Rev. D 76, 121301 (R) (2007); D. Horns and M. Meyer, J. Cosmol. Astropart. Phys. 02 (2012) 033; M. Meyer, D. Horns, and M. Raue, Phys. Rev. D 87, 035027 (2013).

[19] J. P. Conlon and M. C. David Marsh, Phys. Rev. Lett. 111, 151301 (2013); S. Angus, J. P. Conlon, M. C. D. Marsh, A. J. Powell, and L. T. Witkowski, J. Cosmol. Astropart. Phys. 09 (2014) 026.

[20] K. Kohri and H. Kodama, Phys. Rev. D 96, 051701(R) (2017).

[21] T. Moroi, K. Nakayama, and Y. Tang, Phys. Lett. B 783, 301 (2018).

[22] A. Caputo et al., arXiv:1902.02695.

[23] A. G. Dias, A. C. B. Machado, C. C. Nishi, A. Ringwald, and P. Vaudrevange, J. High Energy Phys. 06 (2014) 037; P. W. Graham, I. G. Irastorza, S. K. Lamoreaux, A. Lindner, and K. A. van Bibber, Annu. Rev. Nucl. Part. Sci. 65, 485 (2015); I. G. Irastorza and J. Redondo, Prog. Part. Nucl. Phys. 102, 89 (2018); M. Y. Khlopov, Int. J. Mod. Phys. D 27, 1841013 (2018).

[24] A. C. Melissinos, Phys. Rev. Lett. 102, 202001 (2009).

[25] W. DeRocco and A. Hook, Phys. Rev. D 98, 035021 (2018).

[26] I. Obata, T. Fujita, and Y. Michimura, Phys. Rev. Lett. 121, 161301 (2018).

[27] H. Liu, B. D. Elwood, M. Evans, and J. Thaler, Phys. Rev. D 100, 023548 (2019).

[28] S. M. Carroll, G. B. Field, and R. Jackiw, Phys. Rev. D 41, 1231 (1990); S. M. Carroll, Phys. Rev. Lett. 81, 3067 (1998).

[29] A. Andrianov, D. Espriu, F. Mescia, and A. Renau, Phys. Lett. B 684, 101 (2010); D. Espriu and A. Renau, Phys. Rev. D 85, 025010 (2012); T. Fujita, R. Tazaki, and K. Toma, Phys. Rev. Lett. 122, 191101 (2019).

[30] J. Aasi et al., Classical Quantum Gravity 32, 074001 (2015).

[31] F. Acernese et al., Classical Quantum Gravity 32, 024001 (2015).

[32] K. Somiya, Classical Quantum Gravity 29, 124007 (2012); Y. Aso, Y. Michimura, K. Somiya, M. Ando, O. Miyakawa, T. Sekiguchi, D. Tatsumi, and H. Yamamoto, Phys. Rev. D 88, 043007 (2013); T. Akutsu et al., Prog. Theor. Exp. Phys. (2018), 013F01.

[33] M. Punturo et al., Classical Quantum Gravity 27, 194002 (2010). 
[34] B. P. Abbott et al., Classical Quantum Gravity 34, 044001 (2017).

[35] S. Kawamura et al., J. Phys. Conf. Ser. 120, 032004 (2008).

[36] R. Brito, S. Ghosh, E. Barausse, E. Berti, V. Cardoso, I. Dvorkin, A. Klein, and P. Pani, Phys. Rev. Lett. 119, 131101 (2017); N. Kitajima, J. Soda, and Y. Urakawa, J. Cosmol. Astropart. Phys. 10 (2018) 008; C. S. Machado, W. Ratzinger, P. Schwaller, and B. A. Stefanek, J. High Energy Phys. 01 (2019) 053.

[37] A. Yariv and P. Yeh, Photonics: Optical Electronics in Modern Communications (Oxford University Press, Oxford, 2007).

[38] R. W. P. Drever, J. L. Hall, F. V. Kowalski, J. Hough, G. M. Ford, A. J. Munley, and H. Ward, Appl. Phys. B 31, 97 (1983).

[39] A. J. Millar, G. G. Raffelt, J. Redondo, and F. D. Steffen, J. Cosmol. Astropart. Phys. 01 (2017) 061.

[40] H. J. Kimble, Y. Levin, A. B. Matsko, K. S. Thorne, and S. P. Vyatchanin, Phys. Rev. D 65, 022002 (2001).
[41] D. Budker, P. W. Graham, M. Ledbetter, S. Rajendran, and A. O. Sushkov, Phys. Rev. X 4, 021030 (2014).

[42] R. W. P. Drever et al., Gravitational wave detectors using laser interferometers and optical cavities: Ideas, principles and prospects, in Quantum Optics, Experimental Gravity, and Measurement Theory, edited by P. Meystre and M. O. Scully, NATO Advanced Science Institutes Series (Springer US, Boston, MA, 1983), pp. 503-514.

[43] W. Winkler, A. Rüdiger, R. Schilling, K. Strain, and K. Danzmann, Opt. Commun. 112, 245 (1994).

[44] S. M. Aston et al., Classical Quantum Gravity 29, 235004 (2012).

[45] B. J. Meers, Phys. Rev. D 38, 2317 (1988).

[46] J. Mizuno, K. A. Strain, P. G. Nelson, J. M. Chen, R. Schilling, A. Rüdiger, W. Winkler, and K. Danzmann, Phys. Lett. A 175, 273 (1993).

[47] T. T. Fricke et al., Classical Quantum Gravity 29, 065005 (2012). 